\title{
PRODUCTION AND TRANSPORT OF CONVOY ELECTRONS IN THE CHARGE PREEQUILIBRIUM OF IONS IN SOLIDS ${ }^{(1)}$
}

\author{
P. KOSCHAR, G. SZABÓ*, A. CLOUVAS *, J. KEMMLER, O. HEIL, \\ C. BIEDERMANN, H. ROTHARD, K. KRONEBERGER, S. LENCINAS and \\ K.O. GROENEVELD
}

\begin{abstract}
Institut für Kernphysik, Joh. Wolfg. Goethe-Universität August-Euler-Strasse 6, D-6000 Frankfurt-am-Main 90, F.R.G. * Institute for Nuclear Research of the Hungarian Academy of Sciences (ATOMKI), Pf. 51, H-4001 Debrecen, Hungary * Department of Electrical Engineering, University of Thessaloniki, GR-Thessaloniki, Greece
\end{abstract}

\begin{abstract}
RESUME: Les spectres doublement différentiels, en énergie et en angle, des électrons convoyés produits par des projectiles atomiques légers $\left(\mathrm{H}^{+}, \mathrm{H}^{\circ}, \mathrm{He}^{+}\right)$rapides ( $\left.1.2 \leq \mathrm{E}_{\mathrm{p}} / \mathrm{M}_{\mathrm{p}} \leq 3 \mathrm{MeV} / \mathrm{u}\right)$ transmis à travers des feuilies minces de carbone $\left(2 \leq p x^{-} \leq 20 \mu \mathrm{g} / \mathrm{cm}^{2}\right)$ sont obtenus pour différentes vitesses du projectile et épaisseurs de cible. Dans le domaine hors équilibre de charge, le rendement des électrons convoyés dépend fortement de la charge initiale $q_{i}$ du projectile incident et peut être décrit par un modèle à deux niveaux qui tient compte, d'une part de la production des électrons convoyés dans des procéssus de changement d'état de charge et d'excitation, et d'autre part de leur transport à travers le solide. Les deux différentes structures observées dans les spectres des électrons convoyés peuvent être reproduites par des calculs de distributions de perte d'énergie à l'aide d'une extension du modèle de Landau-Vavilov.
\end{abstract}

ABSTRACT: Absolute double differential convoy electron spectra of light atomic projectiles ( $\left.\mathrm{H}^{+}, \mathrm{H}^{\circ}, \mathrm{He}^{+}\right)\left(1.2 \leq \mathrm{E}_{\mathrm{p}} / \mathrm{M}_{\mathrm{p}} \leq 3 \mathrm{MeV} / \mathrm{u}\right)$ traversing thin carbon foils ( $\left.2 \leq \rho x \leq 20 \mu \mathrm{g} / \mathrm{cm}^{2}\right)$ were measured as function. of the projectile velocity and the target thickness $p x$. In the charge praeequilibrium the convoy electron yield is strongly dependent on the initial charge state $q_{i}$ of the projectile ion and can be described by a two step model which includes the production of convoy electrons as a function of charge changing and excitation processes and their projectile independent transport through the solid. Both the two distinguishable convoy electron structures in the spectra can be represented by calculations of energy loss distributions using an extended Landau-Vavilov model.

At the present time the mechanism of the convoy Electron ( CE) production in solid targets is strongly attributed to charge changing and excitation events of the projectile within the bulk near the surface of the solid. This picture is mainly supported by recent noncoincident and coincident experiments in the projectile charge nonequilibrium regime in thin solids showing a strong dependence of the CE yield on the initial charge state $q_{i}$ of the projectile ion and on the target thickness $\rho x / 1,2 /$. Recently we introduced an extended model of ELC and ECC in solids, which sucessfully described the measured absolute CE yield of the light ion system $\mathrm{H}^{\circ} / \mathrm{H}^{+}(1-3 \mathrm{MeV} / \mathrm{u})$ impact on carbon foils as function of the projectile velocity $v_{p}$ and target thickness ox /1/.

In the present contribution we contihue the discussion about our presented model where we concentrate on an analysis of the shape of the zero degree $C E$ velocity distribution induced by $\mathrm{H}^{\circ}$ and $\mathrm{H}^{+}(3 \mathrm{MeV} / \mathrm{u})$.

\footnotetext{
(1) This work has been funded by the German Federal Minister for Research and Technoloby (BMFT) under the contract number 06 OF 173II, DFG/Bonn; DAAD/Bonn and NATO/Brussels
} 
Because of limited space the reader is refered to ref. $/ 1 /$ for a description of the experimental procedure and data normalisation and for the detailed discussion of the $C E$ yield dependence.

zero degree $C E$ distributions from incident $H^{\circ}$, which carries an initially bound projectile electron, are marked by two distinguishable structures ( see Fig.1.):

- The first is a cusp-iike Convoy Electron Peak ( CEP ) centered at the projectile ion velocity $v_{p}$. The yield dependence / $/$ and shape of the CEP marks it as the true $\mathrm{CE}$ distribution.

- The second structure is a broad distribution with its maximum shifted to velocities below $v_{p}$, named as Inelastic Electron scattering Distribution ( IESD). The resemblance of the IESD with energy loss and angular scattering distributions of isotachic free electrons after traversing solid targets and its strong relationship to the CEP let the IESD be understood as a part of the energy loss spectrum of original $C E$ which are emitted into the small solid angle $\Delta \Omega=(10 \pm 3) * 10^{-6}$ sr of the electron spectrometer.

$C E$ produced deep inside the bulk of the solid as function of the evolution of the charge distribution $F_{j}(x)$ loose their strong space and momentum correlation to the projectile ion by both elastic scattering ( angular straggling) and inelastic scattering (energy loss) with target atoms. The decorrelation can be simply characterized by a transport length $\lambda_{c}$, which is comparable to inelastic mean free paths $\lambda_{e}$ of isotachic free electrons in the case of only week influence of the effective projectile charge. Thus, the CE production and their transport through the solid can be estimated as independent processes. A quantitative treatment of the transport of low energetic electrons ( $\mathrm{E}_{\mathrm{e}}<2000 \mathrm{eV}$ ) is basically aggravated if one considers the high repetition rate of collision events, the high total energy loss compared to $\mathrm{E}_{\mathrm{e}}$, even in thin solid targets used in our experiment, and the increasing influence of discrete collective dipol resonances of the tar-

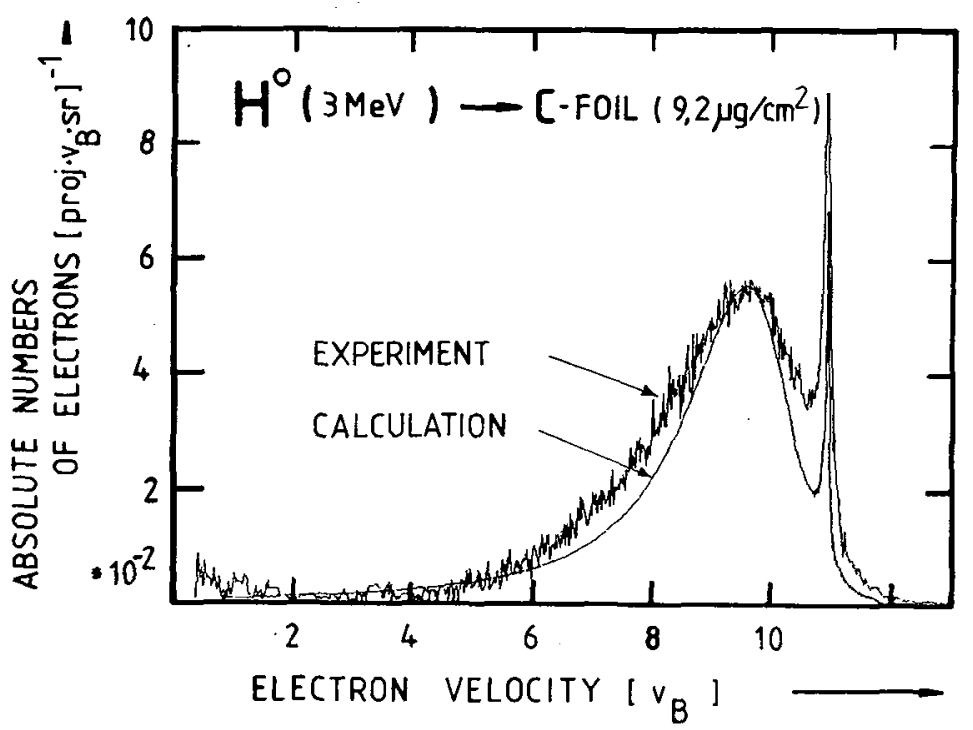

Fig.1. Experimental CE distribution of $\mathrm{H}^{\circ}(3 \mathrm{MeV} / \mathrm{u})$ traversing a carbon foil of $\rho x=(9.2 \pm 0.9) \mu \mathrm{g} / \mathrm{cm}^{2}$ after subtraction of a measured target electron ionisation continuum of $\mathrm{H}+(3 \mathrm{MeV} / \mathrm{u}$ ) impact on the same carbon foil. The overlayed calculated electron distribution with corresponding conditions reproduces the intensity, position and shape of both the IESD and CEP. The differences in the width of the IESD and the intensity of the CEP can be attributed to the experimental error of \pm 108 in the normalisation of the carbon target thickness $\rho x$. 
Fig.2. Three dimensional plot of experimental ( upper part) and calculated (lower part) logarithmic absolute numbers of electrons $\mathrm{r}_{\mathrm{n}}\left(\mathrm{v}_{\mathrm{e}}\right)$ emitted under zero degree from carbon foils after $\mathrm{H}^{\circ}(3 \mathrm{MeV})$ impact as function of the electron velocity $v_{e}$ and the logarithmic target thickness $\rho x$. Both the cusp-like Convoy Electron Peak CE.P at $v=v$ and the Inelastic Electron Scattering Distribution IESD at lowe velocities are quantitatively good reproduced by the calculations (for details see text 1 .

\section{Table 1}

Input data for the calculation of $\mathrm{CE}$ velocity distributions of $\mathrm{H}^{\circ} / \mathrm{H}^{*}$ ( $3 \mathrm{MeV}$ ) deduced from a least square fit to the measured CE yield

$$
\begin{aligned}
& \sigma_{01}\left[10^{-18} \mathrm{~cm}^{2} / \mathrm{at}\right] 18.8 \pm 3 \\
& \sigma_{10}\left[10^{-21} \mathrm{~cm}^{2} / \mathrm{at}\right] \quad 3 \pm 1 \\
& \alpha_{L}^{10}\left[10^{-3}\right] \text { for CEP } 40 \pm 15 \\
& \begin{array}{lll}
\alpha_{C}\left[10^{-3}\right] \text { for } & \text { CEP } & 130 \pm 20 \\
\alpha_{1}\left[10^{-3}\right] \text { for } & \text { IESD } 1.7 \pm .6
\end{array} \\
& \alpha_{C}\left[10^{-3} \text { ]for IESD } 4.5 \pm .8\right. \\
& { }_{\lambda}{ }_{\lambda_{S T}^{C}}^{C}\left[\begin{array}{l}
R \\
R
\end{array}\right] \\
& 16.4 \pm 3 \\
& 160 \pm 10
\end{aligned}
$$

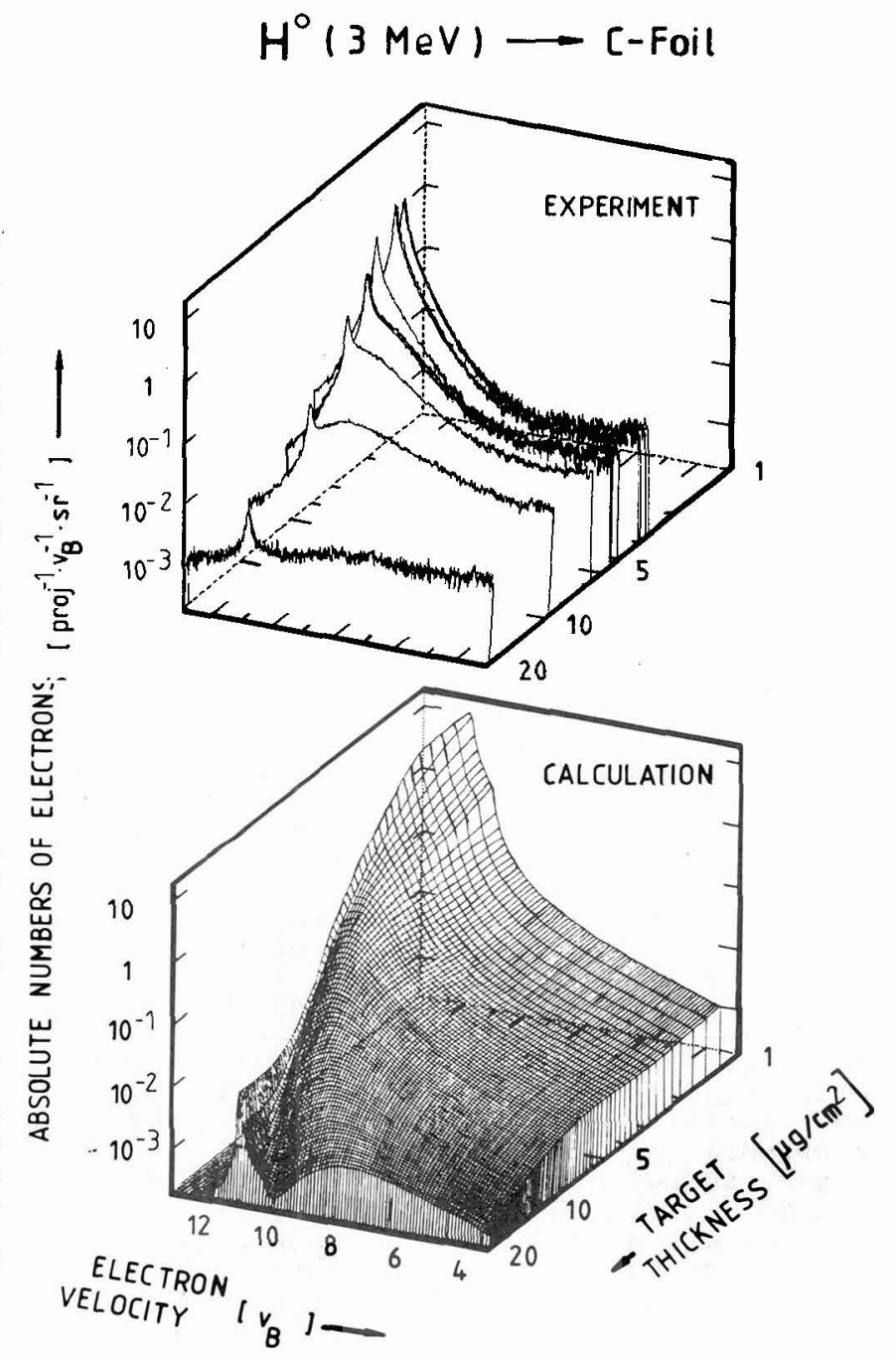

get on the stopping power. For an appropriate modelation of the CEP and the IESD we used for the inelastic contribution a modified Landau-Vavilov model developed for low energetic particles $/ 3 /$, which includes the resonance transitions of the target in the simplified form of an electron gas of constant density with one resonance energy of $\hbar \omega_{0}=25 \mathrm{eV}$ (amorphous carbon). Its applicability is demonstrated by the good agreement between calculations of velocity distributions of monoenergetic free electrons after traversing carbon foils and corresponding experimental results of Jacobi $/ 4 /$ and own measurements within $10 \%\left(1000 \leq E_{e} \leq 2000 \mathrm{eV}\right) / 5 /$. Furthermore, our experimental angular differential spectra show a maintenance of their shape up to large angles of emission which are related to high elastic scattering. This result allows us an elastic scattering independent calculation of CE velocity distributions emitted into a small solid angle $\Delta \Omega$.

In contrary to monoenergetic free electrons CE are continuously produced within the solid target and exhibit a characteristic cusp-like velocity distribution at their place of origin, which is modified by inelastic scattering along their way to the exit surface of the solid ( Landau-Vavilov distribution). The intensity of the CE source inside 
the solid at a position $x$, for both the CEP and IESD, is defined on one hand by the projectile charge distribution $F_{j}(x)$ of $H^{\circ}(j=0)$ and $H^{t}$ $(j=1)$ and by that by the total electron loss and capture cross sections, $\sigma_{01}$ and $\sigma_{10}$, and on the other hand by branching factors, $\alpha_{\tau}$ and $\alpha_{C}$, which describe the constant share of $\mathrm{CE}$ on the total number of lost and captured electrons $/ 1 /$ In case of the CEP the intensity of each contributing slice at $x$ inside the target is diminished by an exponential factor $\exp \left(-\lambda_{C} *\left(x_{0}-x\right)\right.$ ) due to the first scattering event along the distance $x_{0}-x\left(x_{0}\right.$ target thickness $)$. In case of the IESD a similar diminution factor considers the balance of elastic scattering into and out of the solid angle $\Delta \Omega$ of the detector. It is descibed by an effective elastic scattering mean free path $\lambda_{\mathrm{ST}}$, which is, per definition, dependent on the used detector geometry and therefore not directly comparable to elastic mean free paths of free electrons. The zero degree IESD intensity represents only a small section of the angular integrated $C E$ intensity, which mirrors the total CE production inside the solid. Therefore the intensity of the CE source for the IESD is only $3.6 \pm 0.98$ of the corresponding intensity for the CEP.

The shape of each velocity distribution of CE after traversing the distance $x 0^{-x}$ is now determined by the Landau-vavilov distribution. The summation of these spectra of CEP and IESD over the interval $x:=0-x$ results in the velocity distribution of $C E$ at the exit of the foil within the solid angle $\Delta \Omega$.

Fig.1. shows a representative experimental CE distribution of $\mathrm{H}^{\circ}$ ( $3 \mathrm{MeV} / \mathrm{u}$ ) traversing a carbon foil of $\rho x=(9.2 \pm 0.9) \mu \mathrm{g} / \mathrm{cm}^{2}$ overlayed with a calculated electron distribution with corresponding conditions. The quantitative reproduction of the CEP and the IESD is surprisingly good. The differences in the width of the IESD and the intensity of the CEP can be attributed (1) to the error in the target thickness normalisation, (2) to the error in determination of the input data and (3) to the limited validity of the Landau-vavilov model. The extreme sensitivity of the calculation to the input data can be used as a critical test of $\sigma_{01}$ and $\lambda_{C}$ if they are only determined from the CEP intensity as function of $p x$. In tab.1 we summarize our present results for $\mathrm{H}^{\circ} / \mathrm{H}^{+}$which are deduced from least square fits of both the CEP intensity and $C E$ velocity distributions compared to calculations. The complete data set is presented in Fig.2. as a 3-dimensional plot of experimental and calculated data as function of $\rho x$. Shortly summarized we found:

- the shape of the CEP remains cusp-like and nearly symetric comparable to CEP from electron loss in gaseous targets.

- the width only depends on $\lambda_{C}$ and not on $\Delta \Omega$ and increases slightly with decreasing $v_{p}$, due to the increasing stopping power with decreasing $v_{e}$. This is in contrast to the expected linear dependence of the CEP width on $v_{p}$ and $\Delta \Omega$. Furthermore the CEP width is independent on $\rho x$.

- the calculation of CE spectra of $\mathrm{H}^{+}$show similar agreement with experimental data. The absence of the IEsD in this case can be easily understood because the calculated IESD intensity is two orders of magnitudes less than the ionisation electron background.

Further investigations are in progress and will be published in $/ 5 /$.

\section{REFERENCES}

/1/ P.Koschar, A.Clouvas, O.HeiI, M.Burkhard, J.Kemmler K.O.Groeneveld Nucl. Instr. Meth. B24/25 (1987) 153 and ref. therein

/2/ H.P.Hülskötter, J.Burgdörfer, I.A.Sellin Nucl. Instr. Meth. B24/25 (1987) 147 and

J.Kemmler, O.Heil, C.Biedermann, P.Koschar, K.Kroneberger, H.Rothard, K.O.Groeneveld, A.Kövér, Gy.Szabó, D.Berényi, P.Focke, W. Me ckbach

acc. by Lect. Notes in Physics (Springer, 1987) and ref. therein /3/ J.Lindhard, Physica Scripta 32 (1985) 72

/4/ K.Jacobi, Thesis, Technische Universität Clausthal, Germany, 1970

$15 /$ J.Kemmler, S.Lencinas, P.Koschar, O.Heil, H.Rothard, K.0.Groeneveld will be presented at the 12 ICACS in okayama, Japan, oct. 1987 